\title{
Home hospitalisation of exacerbated chronic obstructive pulmonary disease patients
}

\author{
C. Hernandez*, A. Casas*, J. Escarrabill", J. Alonso`, J. Puig-Junoy ${ }^{+}$, E. Farrero", G. Vilagut", \\ B. Collvinent ${ }^{\S}$, R. Rodriguez-Roisin*, J. Roca*, and partners of the CHRONIC project
}

Home hospitalisation of exacerbated chronic obstructive pulmonary disease patients. C. Hernandez, A. Casas, J. Escarrabill, J. Alonso, J. Puig-Junoy, E. Farrero, G. Vilagut, B. Collvinent, R. Rodriguez-Roisin, J. Roca, and partners of the CHRONIC project. C) ERS Journals Ltd 2003.

ABSTRACT: It was postulated that home hospitalisation (HH) of selected chronic obstructive pulmonary disease (COPD) exacerbations admitted at the emergency room (ER) could facilitate a better outcome than conventional hospitalisation.

To this end, 222 COPD patients $(3.2 \%$ female; $71 \pm 10$ yrs (mean \pm SD)) were randomly assigned to $\mathrm{HH}(\mathrm{n}=121)$ or conventional care $(\mathrm{n}=101)$. During $\mathrm{HH}$, integrated care was delivered by a specialised nurse with the patient's free-phone access to the nurse ensured for an 8-week follow-up period.

Mortality (HH: 4.1\%; controls: 6.9\%) and hospital readmissions (HH: $0.24 \pm 0.57$; controls: $0.38 \pm 0.70$ ) were similar in both groups. However, at the end of the follow-up period, HH patients showed: 1$)$ a lower rate of ER visits $(0.13 \pm 0.43$ versus $0.31 \pm 0.62)$; and 2) a noticeable improvement of quality of life ( $\Delta$ St George's Respiratory Questionnaire (SGRQ), -6.9 versus -2.4). Furthermore, a higher percentage of patients had a better knowledge of the disease $(58 \%$ versus $27 \%)$, a better self-management of their condition $(81 \%$ versus $48 \%)$, and the patient's satisfaction was greater. The average overall direct cost per $\mathrm{HH}$ patient was $62 \%$ of the costs of conventional care, essentially due to fewer days of inpatient hospitalisation (1.7 \pm 2.3 versus $4.2 \pm 4.1$ days).

A comprehensive home care intervention in selected chronic obstructive pulmonary disease exacerbations appears as cost effective. The home hospitalisation intervention generates better outcomes at lower costs than conventional care.

Eur Respir J 2003; 21: 58-67.

\begin{abstract}
* Servei de Pneumologia (ICPCT) and Servei d'Urgències, Hospital Clínic, IDIBAPS, ${ }^{*}$ UFISS-Respiratòria (Servei de Pneumologia), Hospital Universitari de Bellvitge Universitat de Barcelona, Health Services Research Unit, Institut Municipal d'Investigació Mèdica (IMIMIMAS) and ${ }^{+}$Research Center for Health and Economics (CRES), Universitat Pompeu Fabra, Barcelona, Spain.
\end{abstract}

Correspondence: J. Roca, Servei de Pneumologia, Hospital Clínic, Villarroel 170, Barcelona 08036, Spain.

Fax: 34932275455

E-mail: jroca@clinic.ub.es

Keywords: Chronic obstructive pulmonary disease, healthcare costs, healthcare services, home care, hospitalisation

Received: February 212002

Accepted after revision: July 262002

Supported by Grants AATM 8/02/99 from the Agencia d'Avaluació de Tecnología Mèdica; FIS 98/0052-01 from the Fondo de Investigaciones Sanitarias; SEPAR 1998; CHRONIC project (IST-1999/12158) from the European Union (DG XIII); and, Comissionat per a Universitats i Recerca de la Generalitat de Catalunya (1999-SGR00228). A. Casas was a predoctoral research fellow supported by CHRONIC and grant-in-aid by ESTEVE group.
Chronic respiratory diseases are an important burden on healthcare systems worldwide [1] that is expected to increase over the forthcoming 2 decades [2], particularly due to chronic obstructive pulmonary disease (COPD). Winter outbreaks of COPD exacerbations mostly occurring in elderly people with concurrent chronic comorbidities often generate dramatic increases in hospital emergency room admissions with subsequent dysfunctions in the healthcare system. It is estimated that hospitalisations of COPD exacerbations represent $\sim 70 \%$ of the overall costs associated with the management of the disease [3].

A first feasibility analysis of home-based services to prevent conventional hospitalisations of COPD exacerbations was reported in 1999 by GRAVIL et al. [4]. Three subsequent controlled trials [5-7] also conducted in the UK have demonstrated both safety and cost reduction when these types of services were applied to selected COPD patients. It is worth noting, however, that none of these studies or the most recent report by SALA et al. [8] showed higher efficacy than conventional hospitalisation in terms of prevention of short-term relapses.

The present investigation was conducted on COPD exacerbations admitted at the emergency room of two tertiary hospitals in the Barcelona area. It was postulated that home hospitalisation with free patient phone access to a specialised nurse should generate a better outcome at lower direct costs than inpatient hospitalisation. Namely: 1) a lower rate of emergency room (ER) relapses; 2) a greater improvement of health-related quality of life (HRQL); and 3) better patient self-management of the disease.

The clinical trial was performed as a preliminary 
step prior to the setting of a technological platform that includes a web-based call centre as one of the core elements [9].

\section{Methods}

\section{Study groups}

Over a 1-yr period (1st November 1999 to 1 st November 2000), 222 patients with COPD exacerbations were included in the study among those admitted at the ER of two tertiary hospitals, Hospital Clínic and Hospital de Bellvitge of Barcelona, Spain. The two primary criteria for inclusion in the study were COPD exacerbation as a major cause of referral to the ER [10] and absence of any criteria for imperative hospitalisation as stated by the British Thoracic Society (BTS) guidelines [11] (i.e., acute chest radiograph changes, acute confusion, impaired level of consciousness, and arterial $\mathrm{pH}<7.35$ ). All COPD exacerbations admitted at the ER on weekdays (Monday to Friday, from 09:00 am to 04:00 pm) during the study period $(n=629)$ were screened by a specialised respiratory team (one chest physician and one nurse) in each hospital. As displayed in the study profile (fig. 1), 220 patients $(35 \%)$ showing one of the following exclusion criteria were not considered candidates for the programme: 1) not living in the healthcare area or admitted from a nursing home
$(11.5 \%, \mathrm{n}=72) ; 2)$ lung cancer and other advanced neoplasms $(5.9 \%, \mathrm{n}=37)$; 3) extremely poor social conditions $(5.2 \%, \mathrm{n}=33) ; 4)$ severe neurological or cardiac comorbidities $(4.8 \%, \mathrm{n}=30) ; 5)$ illiteracy $(4.8 \%$, $\mathrm{n}=30)$; and 6) no phone at home $(2.8 \%, \mathrm{n}=18)$. Onehundred and sixty-five $(26.2 \%)$ of the 629 screened patients required imperative hospitalisation. Up to 244 patients $(38.8 \%)$ were considered eligible for the study, but 22 subjects $(3.5 \%)$ did not sign the informed consent after full explanation of the characteristics of the protocol. The remaining 222 patients $(35.3 \%)$ were blindly assigned using a set of computer-generated random numbers in a 1:1 ratio either to the treatment group (home-based hospitalisation $(\mathrm{HH})$ ) or to the control group (conventional care). One of the hospitals (Hospital Clínic) used a 2:1 randomisation ratio during the first 3 months of the study, which explains the difference in number between the two groups (HH: 121 patients; conventional care: 101 patients).

\section{Home hospitalisation intervention}

Only patients assigned to $\mathrm{HH}$ were assessed by a specialised team. The characteristics of the intervention are summarised in the Appendix. The $\mathrm{HH}$ intervention had three main objectives: 1) an immediate or early discharge from the hospital was encouraged by the specialised team aiming to either avoid or reduce the length of inpatient hospitalisation; 2) a

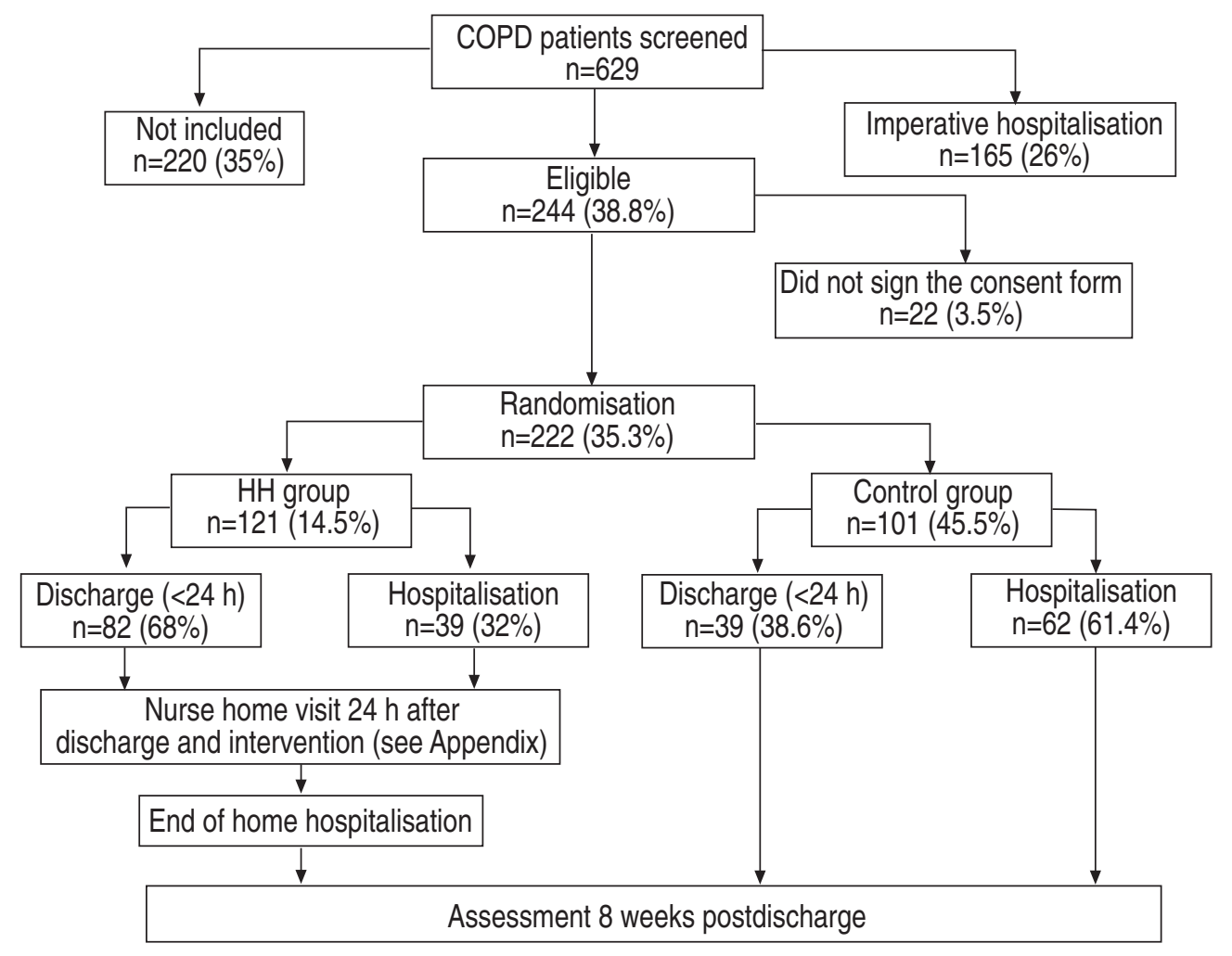

Fig. 1. - Study profile. From the 629 patients screened, $26 \%(\mathrm{n}=165)$ required imperative hospitalisation [11] while up to $35 \%$ (n=220) were not eligible (see text). The remaining 244 patients $(38.8 \%)$ were candidates for the study, but 22 patients $(3.5 \%)$ did not sign the consent form. Two-hundred and twenty-two patients were included (home hospitalisation: 121 and conventional care: 101). COPD: chronic obstructive pulmonary disease; HH: home hospitalisation. 
comprehensive therapeutic approach was tailored on an individual basis, according to the needs detected by the specialised team; and 3) patient support by a skilled respiratory nurse either through home visits or free-phone consultation was ensured during the 8 -week follow-up period. For each $\mathrm{HH}$ patient, a first home visit was scheduled by the nurse within $24 \mathrm{~h}$ after discharge. The length of the home hospitalisation was set by the respiratory nurse. A maximum of five nurse visits at home were permitted during the 8 -week follow-up period, but patient's phone calls to the nurse were not limited in number. The intervention was considered to be a failure if one of the two following events occurred: the patient relapsed and required referral to the ER; or $>5$ nurse visits at home were needed during the follow-up period. In both circumstances, the patients were analysed in the study but they were not considered for a new randomisation (i.e. when attended at the ER for the relapse).

Standard pharmacological treatment was used following COPD guidelines of the Spanish Respiratory Society (SEPAR) [10] during $\mathrm{HH}$ and conventional care. Nonpharmacological interventions for $\mathrm{HH}$ patients, summarised in the Appendix, were performed following specific guidelines [12]. Fragility factors that might facilitate COPD exacerbations were arbitrarily defined by consensus of the research team pre-hoc: 1) severity of pulmonary disease (hypercapnia, cor pulmonale); 2) active comorbidities; 3 ) poor knowledge of the disease; 4) poor compliance with treatment; 5) inadequate skills for the administration of inhaled therapy; 6) low level of social support; and 7) anxiety and/or depression. All of these factors were evaluated both at the initial assessment and then at completion of the follow-up using standard questionnaires, as described below. The response to therapy at home was evaluated by the nurse, based on clinical judgment plus measurements of vital signs and pulse oximetry (Monitor Pulsox ${ }^{\mathrm{TM}}{ }^{3 \mathrm{i}}$; Minolta, AVL Medical Instruments AG, Osaka, Japan). Arterial blood sampling at home for respiratory gases was performed if needed. The nurse's phone access to the physician at the hospital for remote supervision was ensured. Assessment of the progress of the active patients as well as decisions on potential changes in treatment prescription was done during weekly meetings of the specialised team.

\section{Conventional care group}

Patients included in the conventional care group (controls) were evaluated by the attending physician at the ER who decided either on inpatient hospital admission or discharge. Pharmacological prescriptions followed the standard protocols of the centres involved in the study which were similar in the two groups (HH and controls) [10], but the support of a specialised nurse at the ER and at home was not provided for controls. At discharge, the patient was usually supervised by the primary care physician who was not aware of the protocol.
Initial assessment and evaluation 8 weeks after discharge

Initial assessment at admission to the study was identical for both groups patients and included evaluation of the BTS [11] criteria of severity of the exacerbation and blind administration of a questionnaire, described in detail elsewhere [13], about: 1) risk factors for exacerbation (vaccination, smoking habits, comorbidities); 2) HRQL status during the previous year (St George's Respiratory Questionnaire (SGRQ) [14] and Short-Form 12-item survey (SF-12) [15]); 3) history of previous exacerbations (1 yr) requiring inpatient hospitalisations and/or ER admissions evaluated, at least, by questionnaire and, at the most, also by examination of individual clinical records; 4) clinical features of the current exacerbation; 5) fragility factors; and 6) treatment, including compliance, observed skills for administration of inhaled drugs, and rehabilitation at home. Home rehabilitation included interventions, such as manoeuvres to facilitate sputum clearance, nutrition recommendations and skeletal muscle exercise of both upper and lower limbs. Vital signs, chest radiograph films and arterial blood gases were obtained in all patients on admission.

After the 8-week follow-up period, the same questionnaires were administered again to the two groups. In addition, a detailed list of questions on the utilisation of healthcare resources during this period was included. Forced spirometry, chest radiograph films and arterial blood gases were also obtained. A questionnaire to evaluate patient's satisfaction was also blindly administered.

\section{Healthcare costs}

Costs were calculated for each group from the perspective of the public insurer, such that, the cost analysis was restricted to direct healthcare costs. Other resources implied in the programme, such as patient labour time and informal care, were not evaluated in this study.

First, the relevant categories to be considered in order to estimate cost at patient level were identified: 1) length of hospital stay (days of initial hospitalisation plus days during hospital readmissions); 2) ER visits not requiring admission to the hospital; 3) hospital outpatient visits to specialists; 4) primary care physician visits; 5) visits for social support; 6) nurse visits at home; 7) treatment prescriptions; 8) phone calls; and 9) transportation services. Data on use of categories were obtained for each patient during the follow-up period.

A second step was the valuation of resource use. The total cost for each category was calculated as the product of the number of events multiplied by the unit cost per event (i.e. hospitalisation costs were calculated as days in hospital including initial stay plus readmissions multiplied by the average hospitalisation cost per day). Unit costs are expressed as year 2000 prices using Euros $(€)$ as the monetary unit in the European Union. Costs for nurse visits at home, drug 
prescriptions, phone calls and transportation services were directly calculated using information about labour cost, market prices, including value added tax, and overhead costs. Hospital unit costs per hospital stay and visits were not available in the hospitals participating in the study. Instead, average specifically observed tariffs for COPD patients in a public insurance company covering the civil servants of the City Council of Barcelona (PAMEM) were used. These tariffs are mainly paid to public and nonprofit hospitals, and have a close relationship with the real costs. In fact, tariffs represent an adequate basis for cost estimates, given that the present authors' interest is in the financial costs for third party insurers [16].

\section{Statistical analysis}

Results are expressed as mean $\pm \mathrm{SD}$ or as percentages in the corresponding categories. Comparisons between the two study groups on admission and 8 weeks after discharge and changes during the follow-up period were performed using independent t-tests, a nonparametric test (Mann-Whitney U-test) or the Chi-squared test. Changes within each group were assessed using t-test or nonparametric Wilcoxon test for paired samples. Statistical significance was accepted at $\mathrm{p}<0.05$.

\section{Results}

\section{Assessment on first emergency room admission}

Patients of the $\mathrm{HH}$ group and controls showed similar characteristics on ER admission (table 1). HRQL was also similar (SGRQ total score, 58 \pm 17 versus $59 \pm 20, \mathrm{HH}$ and conventional care, respectively; SF-12 physical, $36 \pm 8$ versus $34 \pm 8$; and, SF-12 mental, $44 \pm 12$ versus $44 \pm 13$, respectively). No differences between groups were observed in knowledge of the disease and in self-management of the chronic condition (fig. 2). On average, the two groups showed a relatively acceptable compliance to oral therapy $(79 \%$ of the patients), inhaled therapy (66\%), and long-term oxygen therapy $(82 \%)$. However, they showed poor results in knowledge of the disease (only 20\% of the patients were fully aware of their disorder), appropriate inhalation technique $(26 \%)$, and rehabilitation therapy at home $(10 \%)$. Forced spirometric measurements at week 8 after discharge did not show differences between the two groups (table 1).

\section{Outcomes}

Five patients $(4.1 \%)$ in the HH group and 7 controls $(6.9 \%)$ died during the 8-week follow-up period (table 2). The rate of hospital readmissions during this period was $\sim 25 \%$, with no differences between

Table 1.-Baseline characteristics of the study groups

\begin{tabular}{|c|c|c|c|}
\hline & Home hospitalisation & Conventional Care & Total \\
\hline Subjects n (\% female $)$ & $121(3.3)$ & $101(3.0)$ & $222(3.2)$ \\
\hline Age yrs & $71.0 \pm 9.9$ & $70.5 \pm 9.4$ & $70.8 \pm 9.7$ \\
\hline Respiratory rate $\cdot \min ^{-1}$ & $26.9 \pm 6.0$ & $26.8 \pm 5.9$ & $26.8 \pm 5.9$ \\
\hline Dyspnoea score (VAS) & $6.1 \pm 3.1$ & $6.2 \pm 3.3$ & $6.2 \pm 3.2$ \\
\hline \multicolumn{4}{|l|}{ Risk factors } \\
\hline Influenza vaccination \% & 66.1 & 65.3 & 65.8 \\
\hline Current smokers $\%$ & 27.3 & 17.8 & 23.0 \\
\hline Comorbidities \% & 93.4 & 96.0 & 94.6 \\
\hline Number of comorbid conditions & $2.9 \pm 1.8$ & $3.1 \pm 1.6$ & $3.1 \pm 1.7$ \\
\hline \multicolumn{4}{|l|}{$\begin{array}{l}\text { Exacerbations requiring in-hospital admission } \\
\text { (previous year) }\end{array}$} \\
\hline Subjects \% & 40.8 & 40.6 & 40.7 \\
\hline Number of episodes & $0.7 \pm 1.2$ & $0.9 \pm 1.4$ & $0.8 \pm 1.2$ \\
\hline \multicolumn{4}{|l|}{ Oxygen therapy at home } \\
\hline Patients \% & 12.4 & 18.8 & 15.3 \\
\hline \multicolumn{4}{|l|}{ Arterial blood gases (on admission) } \\
\hline$F \mathrm{I}, \mathrm{O}_{2}$ & $21.7 \pm 1.4$ & $22.1 \pm 2.3$ & $21.9 \pm 1.8$ \\
\hline $\mathrm{pH}$ & $7.4 \pm 0.04$ & $7.4 \pm 0.3$ & $7.4 \pm 0.2$ \\
\hline $\mathrm{Pa}, \mathrm{O}_{2}$ & $65.0 \pm 13.6$ & $64.7 \pm 16.4$ & $64.9 \pm 14.9$ \\
\hline $\mathrm{Pa}_{\mathrm{a}, \mathrm{CO}_{2}}$ & $42.7 \pm 7.5$ & $43.8 \pm 8.9$ & $43.2 \pm 8.2$ \\
\hline Blood sampling at $F \mathrm{I}, \mathrm{O}_{2}=0.21 \%$ patients & 77.6 & 72.6 & 75.4 \\
\hline$P \mathrm{a}, \mathrm{O}_{2}$ breathing $F \mathrm{I}_{1} \mathrm{O}_{2}=0.21$ & $63.2 \pm 10.5$ & $62.9 \pm 13.9$ & $63.1 \pm 12.1$ \\
\hline \multicolumn{4}{|l|}{ Forced spirometry (at 8 weeks of follow-up) } \\
\hline FVC L ( $\%$ pred $)$ & $2.4 \pm 0.9(64)$ & $2.2 \pm 0.9(60)$ & $2.3 \pm 0.9(62)$ \\
\hline FEV1 L (\% pred) & $1.2 \pm 0.6(43)$ & $1.1 \pm 0.4(41)$ & $1.1 \pm 0.5(42)$ \\
\hline $\mathrm{FEV} 1 / \mathrm{FVC} \%$ & $50 \pm 13.3$ & $50 \pm 13.1$ & $50 \pm 13.2$ \\
\hline
\end{tabular}

Results are expressed either as mean \pm SD or as a percentage of subjects in the corresponding category. Total: combined data of the two groups; VAS: visual analogue scale for scoring dyspnoea; $F \mathrm{I}, \mathrm{O}_{2}$ : inspiratory oxygen fraction; $\mathrm{pH}$ : arterial $\mathrm{pH} ; P \mathrm{a}, \mathrm{O}_{2}$ : oxygen tension in arterial blood; $\mathrm{Pa}_{\mathrm{a}, \mathrm{CO}_{2}}$ : carbon dioxide tension in arterial blood; FVC: forced vital capacity; pred: predicted; FEV1: forced expiratory volume in one second; FEV1/FVC: ratio, expressed as an actual value. 


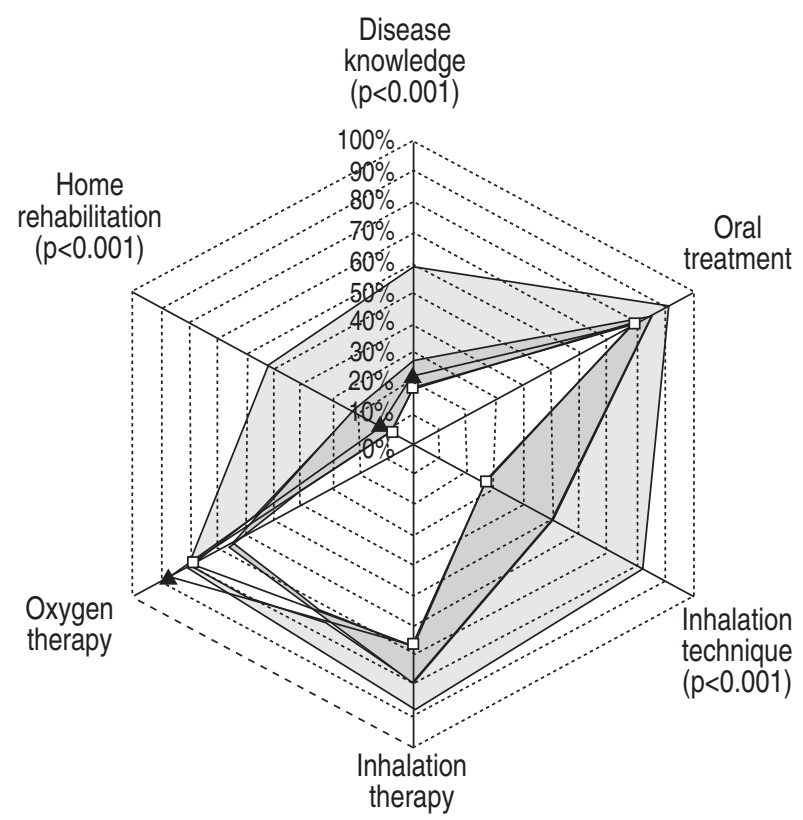

Fig. 2.-Knowledge of the disease and self-management of the chronic condition. Results are expressed as per cent of patients. On admission (inner limits: home hospitalisation (HH): controls: $\square$ ), no differences were seen in any of the six dimensions of the graph. No changes in the control group (dark grey area) were seen during the 8 -week follow-up period, but marked beneficial effects were detected in the HH group (light grey area). groups. In the control group, however, the rate of relapses requiring new ER admission without subsequent hospital readmissions almost doubled the figure shown by the $\mathrm{HH}$ patients $(\mathrm{p}<0.05)$. As indicated in table 2, the HH group showed higher improvement in HRQL and higher satisfaction than the control group after the 8-week follow-up period. Furthermore, a higher percentage of patients in the $\mathrm{HH}$ group (fig. 2) had a substantial improvement in knowledge of the disease $(\mathrm{HH} 58 \%$ versus $27 \%$ for controls, $\mathrm{p}<0.01)$, compliance on inhalation technique $(\mathrm{HH} 81 \%$ versus $48 \%$ for controls, $\mathrm{p}<0.001$ ), and rehabilitation at home ( $\mathrm{HH} 51 \%$ versus $21 \%$ for controls, $\mathrm{p}<0.01$ ).

\section{Characteristics of inpatient hospitalisation}

Up to $68 \%$ of $\mathrm{HH}$ patients were discharged from ER without requiring hospitalisation $(<24 \mathrm{~h})$ compared to $39 \%$ of the control patients $(\mathrm{p}<0.001$; table 2). Consequently, the length of hospitalisation was also significantly lower in the $\mathrm{HH}$ group than in controls (1.7 versus 4.2 days, respectively; $\mathrm{p}<0.001)$. Hospitalisation for $>3$ days was required in $48 \%$ of controls but only $17 \%$ of $\mathrm{HH}$ patients. Characteristics of inpatient hospitalisation in the two groups are reported in table 2 . The average length of the stay in the hospitalised patients of the control group was 8.1 days.

Table 2. - Main outcomes of the study and characteristics of the initial inpatient hospitalisation

\begin{tabular}{|c|c|c|c|}
\hline & Home hospitalisation & Conventional care & p-value \\
\hline \multicolumn{4}{|c|}{ Clinical outcomes (8-week follow-up) } \\
\hline \multicolumn{4}{|c|}{ Inpatient hospital readmissions } \\
\hline Patients n $(\%)$ & $23(20.0)$ & $26(27.7)$ & \\
\hline Number of episodes & $0.24 \pm 0.57$ & $0.38 \pm 0.70$ & \\
\hline \multicolumn{4}{|c|}{ Emergency room readmissions } \\
\hline Patients n $(\%)$ & $11(9.6)$ & $21(22.3)$ & $0.02^{\#}$ \\
\hline Number of episodes & $0.13 \pm 0.43$ & $0.31 \pm 0.62$ & $0.01^{\top}$ \\
\hline Deaths n $(\%)$ & $5(4.1)$ & $7(6.9)$ & \\
\hline \multicolumn{4}{|c|}{ Health-related quality of life ( 8 -week follow-up) } \\
\hline \multicolumn{4}{|c|}{ Mean $\triangle$ SGRQ score } \\
\hline Total & -6.9 & -2.4 & $0.05^{+}$ \\
\hline Symptoms & -8.7 & -8.4 & \\
\hline Activity & -4.8 & -0.09 & \\
\hline Impact & -7.6 & -1.9 & $0.03^{+}$ \\
\hline \multicolumn{4}{|l|}{ Mean $\Delta$ SF-12 score } \\
\hline Physical & 1.7 & 1.9 & \\
\hline Mental & 2.0 & -0.05 & \\
\hline \multicolumn{4}{|l|}{ Patient's satisfaction } \\
\hline Mean score & 8.0 & 7.5 & $0.03^{\oplus}$ \\
\hline \multirow{2}{*}{\multicolumn{4}{|c|}{ Inpatient hospitalisation }} \\
\hline & & & $\%$ of patients hospitalised \\
\hline$<1$ day $\%$ & 67.8 & 38.6 & $<0.001^{\#}$ \\
\hline 2 days $\%$ & 5.8 & 4.0 & \\
\hline 3 days $\%$ & 9.9 & 9.9 & \\
\hline$>3$ days $\%$ & 16.5 & 47.5 & \\
\hline \multirow[t]{2}{*}{ Days of hospitalisation } & $1.71 \pm 2.33$ & $4.15 \pm 4.10$ & $<0.001^{\bullet}$ \\
\hline & {$[0-11]$} & {$[0-16]$} & \\
\hline
\end{tabular}

Results are expressed either as mean $\pm \mathrm{SD}$ or as a percentage of subjects in the corresponding category. Minimum and maximum values are expressed in square brackets. SGRQ: St George's Respiratory Questionnaire; SF-12: Short-Form 12item survey. ${ }^{\#}$ : Chi-squared test; ${ }^{\natural}$ : Mann-Whitney U nonparametric test for independent samples; ${ }^{+}$: t-test for comparison of two independent samples. 
In the $\mathrm{HH}$ group, the average length of the homebased hospitalisation was 3.56 days (1-14 days). During the 8-week follow-up period, the number of nurse visits at home was $1.66 \pm 1.03$ (range, $0-4$ ) and the number of nurse phone calls to patients was $1.56 \pm 1.31(0-6)$. Likewise, the number of patients' phone calls to the nurse was $0.76 \pm 1.34(0-9)$, such that the overall number of phone calls was $2.33 \pm 2.05$ (0-10).

As indicated in table 3, the control group showed a higher average cost per patient than the HH group in terms of length of hospitalisation and ER visits. Conversely, the control group displayed lower costs for prescription than $\mathrm{HH}$. During the follow-up period, no differences between the two groups were seen in the use of the following three categories: visits to primary care physician, transportation, and social support. The average overall healthcare cost per patient in the $\mathrm{HH}$ group was only $62 \%$ of the average cost calculated for control patients $(€ 1,255$ versus $€ 2,033 ; \mathrm{p}=0.003)$.

\section{Discussion}

The present study indicates that home hospitalisation as described in the Appendix generated better outcomes than conventional care of COPD exacerbations. Better outcomes with $\mathrm{HH}$ included: 1) lower hospitalisation rates; 2) lower rates of short-term relapses requiring ER admissions; 3) clinically relevant improvement in HRQL, as assessed by the SGRQ [17]; 4) a higher degree of patient satisfaction; and 5) an important positive impact on knowledge of the disease and on patient self-management of the chronic condition.
The results were obtained with a rather modest use of the resources allocated to home support. Only a small portion of the five potential nurse visits was used (on average 1.7 nurse visits at home) during the 2-month follow-up period. Despite the free-phone access that was ensured to all patients, the average number of patients' phone calls to the nurse was only 0.76 . Somewhat unexpectedly, the study shows that home hospitalisation was less costly than conventional care. The average overall costs per $\mathrm{HH}$ patient were substantially lower than in conventional care, essentially due to fewer days of inpatient hospitalisation. Slightly higher costs in the $\mathrm{HH}$ group were only observed in prescriptions that were due to both oxygen therapy and nebuliser therapy, because these two treatments were part of the inpatient hospitalisation costs in a substantial portion of the control group.

While all previous studies assessing either home hospitalisation or early discharge [5-8] have essentially shown that the approach is safe, this is the first report that clearly demonstrates the beneficial effects of the intervention compared with conventional care of COPD exacerbations. The present study also indicates that improvement of the outcomes can be associated with a reduction of direct costs. Like other reports [5-8], the present study confirms that home hospitalisation is suitable only in a subset of exacerbations that must be selected at the hospital after proper assessment by a specialised team.

\section{Internal validity of the trial}

The validity of the assignment process for either $\mathrm{HH}$ or conventional care was ensured by both the generation of the allocation sequence by a random

Table 3. - Average direct cost per patient for the two study groups

\begin{tabular}{|c|c|c|c|c|c|c|}
\hline \multirow[t]{2}{*}{ Categories } & \multirow{2}{*}{$\begin{array}{l}\text { Costs per } \\
\text { category } \\
€ €\end{array}$} & \multicolumn{2}{|c|}{ Home hospitalisation } & \multicolumn{2}{|c|}{ Conventional care } & \multirow[t]{2}{*}{ p-value $\mathrm{e}^{\#}$} \\
\hline & & $\begin{array}{l}\text { No. of events/ } \\
\text { patients }\end{array}$ & $\begin{array}{l}\text { Cost per } \\
\text { patient } €\end{array}$ & $\begin{array}{l}\text { No. of events/ } \\
\text { patients }\end{array}$ & $\begin{array}{l}\text { Cost per } \\
\text { patient } €\end{array}$ & \\
\hline Inpatient hospital stay & 220.62 & $495 / 77$ & 941.40 & $765 / 81$ & 1795.47 & $<0.001$ \\
\hline ER visits & 79.71 & $15 / 11$ & 10.31 & $29 / 21$ & 24.59 & 0.01 \\
\hline Outpatient visits & 39.85 & $16 / 12$ & 5.49 & $52 / 14$ & 22.04 & \\
\hline Primary care physician visits & 47.48 & $20 / 6$ & 8.19 & $15 / 8$ & 7.57 & \\
\hline Social support visits & 18.75 & $10 / 3$ & 1.62 & $11 / 4$ & 2.19 & \\
\hline Nurse home visit & 25.34 & 192/101 & 41.94 & & & \\
\hline Prescriptions & & & 217.21 & & 172.06 & 0.001 \\
\hline \multicolumn{7}{|l|}{ Phone calls: } \\
\hline Patient to nurse & & $88 / 46$ & & & & \\
\hline Nurse to patient & & $182 / 96$ & & & & \\
\hline Total & 9.02 & $270 / 99$ & 20.99 & & & \\
\hline Transport & 6.01 & $154 / 77$ & 7.97 & $150 / 61$ & 9.59 & \\
\hline $\begin{array}{l}\text { Average direct cost } \\
\text { per patient }(95 \% \mathrm{CI})\end{array}$ & & & $\begin{array}{c}1255.12 \\
(978.54-1568.04)\end{array}$ & & $\begin{array}{c}2033.51 \\
(1547.05-2556.81)\end{array}$ & 0.003 \\
\hline
\end{tabular}


process and preventing any foreknowledge of the treatment assignments by the specialised team that implemented the allocation sequence [18]. As described in the Methods section, one of the hospitals (Hospital Clínic) transiently used a 2:1 randomisation ratio as a conservative approach to ensure an adequate number of $\mathrm{HH}$ patients. This strategy provoked a lack of equilibrium in the number of patients assigned to each group (HH: 121; controls: 101), but does not seem to compromise the comparability between the two groups, as shown by the similar results obtained in the assessment on admission.

Since missing data represented $<2 \%$ of the study group, it can be considered that the aims of the followup analysis were fully achieved. It is worth noting, however, that the relatively short follow-up planned in the study might have reduced the impact of the positive effects shown by educational intervention (fig. 2). The pivotal effects of education on selfmanagement of asthma have been widely demonstrated in recent years $[19,20]$ and evidence of this has recently been reported for COPD patients [21]. The present study identifies this area as a key field for the development of future guidelines for chronic respiratory diseases.

In the economical analysis, the limitation of selfreported use of healthcare resources was partially palliated by the evaluation of the clinical records of the patients. An excellent correlation between the two scores was observed. The economic evaluation performed in the context of this randomised controlled trial was designed to ask the following question: does substituting hospital-at-home care for hospital care in COPD exacerbations result in a lower cost to the health service?

This economic evaluation may be affected by two main limitations. First, the perspective of the evaluation was that of the public healthcare insurer, excluding nonhealthcare costs. In this study, formal (paid work) or informal (unpaid work and leisure time) care for exacerbated COPD patients were not evaluated. Notwithstanding, a previous randomised controlled trial comparing hospital-at-home care with inpatient care [22] reported that carers' expenses made up a small proportion of total costs and inclusion of these costs did not alter the results.

A second limitation of the economic evaluation is that average costs were used to evaluate hospital care. In fact, hospital resources released for the care of other patients may be less than the final average cost when patients are nearing the end of their hospital stay and therefore require less resource intensity (marginal cost). It has been argued that the existence of fixed hospital costs amplifies the value of any potential savings resulting from a reduction in beddays [23].

However, marginal costs estimated as the short-run variable costs are not appropriate to evaluate the costs (or savings) that would be associated with the provision of new hospital services in the long-term [24]. From the theoretical point of view, average costs may appropriately represent the value of freed resources, assuming that patients can be admitted to empty beds. Even so, a sensitivity analysis was performed assuming that resources released by home hospitalisation intervention (days of hospital) would be either $75 \%$ or $50 \%$ of the average cost. Under both assumptions, it was found that the average cost per patient in the $\mathrm{HH}$ group was lower than the cost calculated for control patients. It may therefore be asserted that using marginal cost to evaluate resources does not result in home hospitalisation being more costly than conventional care for exacerbated COPD patients.

\section{External validity}

The positive outcomes obtained in the study probably reflect the combined effects of the comprehensive home care intervention (Appendix) undertaken in this trial. It is worth noting, however, that while the reduction of ER readmissions in the $\mathrm{HH}$ group was clear, the impact on short-term hospital readmissions was rather modest, as seen in other reports [5].

It is remarkable that the results of the present study fully substantiate and amplify the message given by studies [5,6] carried out in the UK, despite noticeable country differences in terms of interactions between primary care and tertiary hospitals. While in Barcelona, $\sim 70 \%$ of the ER admissions in tertiary hospitals for COPD exacerbations corresponded with self-referrals [25]. This figure falls to $\sim 30 \%$ on average in the UK and as low as $1 \%$ in the report by SKWARSKA et al. [5]. The present results seem to support the notion that the efficacy of $\mathrm{HH}$ is not dependent on the specificities of the healthcare system if the logistics of the home care services are fully managed by the hospital. Whether this type of setting should be recommended or not is still controversial. Alternatively, a distributed model based on a close collaboration between healthcare levels [26, 27] has been suggested, as discussed below.

Although the current investigation purposely followed general aspects of the study profile reported by SKWARSKA et al. [5], a proper comparative analysis between the two studies is difficult because of several factors. First, differences in the healthcare systems are not negligible as alluded to above. Second, the Scottish patients were randomised after ER doctors had already decided on hospital admission, which was not the case in the present study. This factor might have resulted in a selection of more severe patients in the two groups (HH and controls) in [5] as compared to the present study. It can be speculated that the clear beneficial effects described in the present investigation (not seen in [5]) might be because patients in Barcelona had less severe exacerbations. It is worth noting that UK studies [4-7] on different modalities of home hospitalisation consistently showed, on average, lower FEV1, higher SGRQ scores and lower rates of autoreferrals to ERs of tertiary hospitals than in studies carried out in Spain [8, 13], suggesting that sicker patients were attending in UK hospitals. This is probably due to country differences in the interactions between healthcare levels.

It can be concluded, however, that an assignment bias was not present in these two studies. Moreover, the patients of the present study showed similar characteristics to those reported by studies on 
exacerbated COPD patients admitted in the ER of tertiary hospitals in Spain $[8,13]$.

There is controversy regarding the effects of hospitalat-home schemes on costs for COPD patients. Two randomised controlled trials [22, 23] reported that hospital-at-home significantly increased healthcare costs for COPD patients. The two trials, however, analysed a very small sample of patients whose severity of illness was not delineated.

The economic evaluation of home hospitalisation in the current study clearly reported cost savings. As stated in the Results section, savings may be mainly attributed to the reduction in the length of stay for patients in the $\mathrm{HH}$ programme. The magnitude of this reduction in the present study is enough to compensate the increase in the costs corresponding to the $\mathrm{HH}$ programme. In this sense, the present results confirm the importance of the impact of the intervention on the use of this resource for COPD patients in the economic evaluation of home care programmes as the sensitivity analysis of SHEPPERD et al. [22] indicated.

\section{Implications for healthcare policy}

The search for healthcare services meeting the needs of chronically ill people [26, 27] has recently generated the so-called chronic care model [28]. These authors propose a patient-centred approach, with special emphasis on shared care arrangements across the healthcare system (between specialised care at the hospital and primary care) and within the multidisciplinary primary care team. Key features of the model [28] are the development of innovative homebased services with involvement of patients (and caregivers) as partners in the management of the disease.

A key challenge in the development of such new services is a redefinition of the roles and skills of the specialised nurses and physiotherapists [29, 30]. The interactions of these allied healthcare professionals with physicians should be re-examined. Adequate standardisation of procedures is also needed. In this new setting, there is an important role for the use of information technologies, facilitating the interactions between healthcare levels and the development of novel educational tools.

It can be concluded that home hospitalisation of selected chronic obstructive pulmonary disease exacerbations generates better outcomes at lower costs than conventional care. The data of the present study suggests that managerial aspects of exacerbated chronic obstructive pulmonary disease patients must be revisited. Home-based services (home hospitalisation or home support) should be taken as part of the continuum of care in chronically ill patients. Despite the promising results of these new approaches in the treatment of chronic obstructive pulmonary disease exacerbations, prevention of early relapses after discharge is still an important challenge. The present study prompts the need for the deployment of this type of intervention as a regular healthcare service for exacerbated chronic obstructive pulmonary disease patients under the frame of a properly designed costeffectiveness analysis.
Appendix: Description of the intervention in the home hospitalisation group

Assessment on ER admission by the specialised team

1. Characteristics of the exacerbation, comorbidities, and response to treatment at the ER

1.1. Baseline conditions of the patient (duration $1.5 \mathrm{~h})$ : a) health-related quality of life; b) healthcare resources in the previous year; c) fragility risk factors; and knowledge of the disease and compliance to therapy.

1.2. Decision on discharge from the ER or after a short period of inpatient hospitalisation based on 1.1. and 1.2.

\section{Treatment at discharge}

2.1 Pharmacological therapy of COPD and comorbidities

2.2. Nonpharmacological treatment (duration $2 \mathrm{~h}$ ): a) education on knowledge of the disease; adherence to treatment; and recognition/prevention of triggers of exacerbation; b) selection of appropriate equipment at home; training on administration of pharmacological treatment; c) smoking cessation; d) patient empowerment on daily life activities: hygiene, dressing, household tasks; leisure activities; breathing exercises; and, skeletal muscle activity; e) nutrition recommendations; and f) socialisation and changes in lifestyle.

3. Home hospitalisation and 8-week follow-up

3.1. First nurse visit at home at $24 \mathrm{~h}$ (duration $1 \mathrm{~h}$ ) a) Assessment of the response to pharmacological treatment

b) Introduction of changes under remote physician's supervision

c) On-site assessment of fragility factors

d) Action plan revisited and education reinforced

3.2. Eight-week follow-up

a) Number of home visits and duration of $\mathrm{HH}$ were decided by the nurse

b) Patient free-phone access to the nurse was ensured

c) Nurse phone calls to patient to reinforce the action plan

3.3. Failure of the programme

a) More than five nurse home visits during the 8-week follow-up

b) New problem requiring ER admission

4. Assessment after 8-week follow-up (see text)

Acknowledgements. The authors are grateful to A. Alonso, J.A. Barberà, E. Sala, E. Gavela, and the nurses I. Fernandez and 
C. Fornas, from the Hospital Clínic (Barcelona, Spain) for their support during the study and in the preparation of the manuscript. The authors also acknowledge the support of $\mathrm{N}$. Celorrio and nurse M. Maderal from the Hospital de Bellvitge (Barcelona, Spain) for their valuable contribution. The authors are indebted to R. Santed from the IMIM for his job in the preparation of the database, and thank J.M. Marín, from the Hospital Miguel Servet (Zaragoza, Spain) for his collaboration in the design of the protocol. Finally, the authors are grateful to all of the following partners of the CHRONIC project. CSC, Barcelona: A. Alonso, J.A. Montero, J. Pastor and B. Sifre; UPM, Madrid, Spain: F. del Pozo, P. de Toledo, S. Jiménez; CESTEL, Madrid: F. Ortiz, L. Mena, M. Godoy, J. Luís Sirera; CSIC-CNM, Barcelona: J. Aguiló, L. Sánchez, A. Guimerà; SMS, Milano, Italy: A. Falco, D. Balconi; SMS, Madrid: B. Andrés, J.J. Moratillas; ICP, Milano: A. Mattiussi, A. Antonini (Parkinson Center); MC, Milano: C. Castiglioni, E. Battaglia; TILAB, Turín, Italy: F. Moggio, M. Mercinelli; KUL, Leuven, Belgium: M. Decramer, T. Troosters, W. Droogne, F. Van de Werf; EW, Paris, France: P. Sylvester, N. Pougetoux; ALAMO, Madrid: M.A. García Matatoros, A. Marcus; UB, Barcelona: R. Farré; ECOMIT, Barcelona: C. Céinos.

\section{References}

1. Pauwels RA, Buist AS, Calverley PM, Jenkins CR, Hurd SS. Global strategy for the diagnosis, management, and prevention of chronic obstructive pulmonary disease. NHLBI/WHO Global Initiative for Chronic Obstructive Lung Disease (GOLD) Workshop summary. Am J Respir Crit Care Med 2001; 163: 1256-1276.

2. Murray CJ, Lopez AD. Global mortality, disability, and the contribution of risk factors: Global Burden of Disease Study. Lancet 1997; 349: 1436-1442.

3. Strassels SA, Smith DH, Sullivan SD, Mahajan PS. The costs of treating COPD in the United States. Chest 2001; 119: 344-352.

4. Gravil JH, Al Rawas OA, Cotton MM, Flanigan U, Irwin A, Stevenson RD. Home treatment of exacerbations of chronic obstructive pulmonary disease by an acute respiratory assessment service. Lancet 1998; 351: 1853-1855.

5. Skwarska E, Cohen G, Skwarski KM, et al. Randomized controlled trial of supported discharge in patients with exacerbations of chronic obstructive pulmonary disease. Thorax 2000; 55: 907-912.

6. Cotton MM, Bucknall CE, Dagg KD, et al. Early discharge for patients with exacerbations of chronic obstructive pulmonary disease: a randomized controlled trial. Thorax 2000; 55: 902-906.

7. Davies L, Wilkinson M, Bonner S, Calverley PM, Angus RM. "Hospital at home" versus hospital care in patients with exacerbations of chronic obstructive pulmonary disease: prospective randomized controlled trial. BMJ 2000; 321: 1265-1268.

8. Sala E, Alegre L, Carrera M, et al. Supported discharge shortens hospital stay in patients hospitalized because of an exacerbation of COPD. Eur Respir $J$ 2001; 17: 1138-1142.

9. CHRONIC project. An information capture and processing environment for chronic patients in the information society. V program of the European Union, DG XIII, IST-1999/12158 (technical annex 1999, internal document). http://chronic.cestel.es. Date last accessed: February 2002.

10. Barbera JA, Peces-Barba G, Agusti AG, et al. Clinical guidelines for the diagnosis and treatment of chronic obstructive pulmonary disease. Arch Bronconeumol 2001; 37: 297-316.

11. BTS guidelines for the management of chronic obstructive pulmonary disease. The COPD Guidelines Group of the Standards of Care Committee of the BTS. Thorax 1997; 52: Suppl. 5, S1-S28.

12. Giner J, Basualdo LV, Casan P, et al. [Guideline for the use of inhaled drugs. The Working Group of SEPAR: the Nursing Area of the Sociedad Espanola de Neumologia y Cirugia Toracica]. Arch Bronconeumol 2000; 36: 34-43.

13. Garcia-Aymerich J, Monso E, Marrades RM, et al. Risk factors for hospitalization for a chronic obstructive pulmonary disease exacerbation. EFRAM study. Am J Respir Crit Care Med 2001; 164: 1002-1007.

14. Ferrer M, Alonso J, Prieto L, et al. Validity and reliability of the St George's Respiratory Questionnaire after adaptation to a different language and culture: the Spanish example. Eur Respir J 1996; 9: $1160-1166$.

15. Ware J Jr, Kosinski M, Keller SD. A 12-Item ShortForm Health Survey: construction of scales and preliminary tests of reliability and validity. Med Care 1996; 34: 220-233.

16. Drummond $\mathrm{M}, \mathrm{McGuire} \mathrm{A}$. Economic evaluation in health care. Merging theory and practice. London, Office of health Economics, 2001.

17. Jones PW. Issues concerning health-related quality of life in COPD. Chest 1995; 107: Suppl. 5, 187S-193S.

18. Moher D, Schulz KF, Altman DG, Lepage L. The CONSORT statement: revised recommendations for improving the quality of reports of parallel-group randomized trials. Lancet 2001; 357: 1191-1194.

19. Brooks CM, Richards JM, Kohler CL, et al. Assessing adherence to asthma medication and inhaler regimens: a psychometric analysis of adult self-report scales. Med Care 1994; 32: 298-307.

20. Gallefoss F, Bakke PS. How does patient education and self-management among asthmatics and patients with chronic obstructive pulmonary disease affect medication? Am J Respir Crit Care Med 1999; 160: 2000-2005.

21. Watson PB, Town GI, Holbrook N, Dwan C, Toop LJ, Drennan CJ. Evaluation of a self-management plan for chronic obstructive pulmonary disease. Eur Respir J 1997; 10: 1267-1271.

22. Shepperd S, Harwood D, Gray A, Vessey M, Morgan P. Randomized controlled trial comparing hospital at home care with inpatient hospital care. II: cost minimization analysis. BMJ 1998; 316: 1791-1796.

23. Ruchlin HS, Dasbach EJ. An economic overview of chronic obstructive pulmonary disease. Pharmacoeconomics 2001; 19: 623-642.

24. Coast J, Richards SH, Peters TJ, Gunnell DJ, Darlow MA, Pounsford J. Hospital at home or acute hospital care? A cost minimization analysis. BMJ 1998; 316: 1802-1806. 
25. Garcia-Aymerich J, Barreiro E, Farrero E, Marrades RM, Morera J, Anto JM. Patients hospitalized for COPD have a high prevalence of modifiable risk factors for exacerbation (EFRAM study). Eur Respir $J$ 2000; 16: 1037-1042.

26. Wagner $\mathrm{EH}$. The role of patient care teams in chronic disease management. BMJ 2000; 320: 569-572.

27. Wagner EH. Meeting the needs of chronically ill people. BMJ 2001; 323: 945-946.

28. Wagner EH, Glasgow RE, Davis C, et al. Quality improvement in chronic illness care: a collaborative approach. Jt Comm J Qual Improv 2001; 27: 63-80.

29. Farrero E, Escarrabill J, Prats E, Maderal M, Manresa F. Impact of a hospital-based home-care program on the management of COPD patients receiving long-term oxygen therapy. Chest 2001; 119: 364-369.

30. Mundinger MO, Kane RL, Lenz ER, et al. Primary care outcomes in patients treated by nurse practitioners or physicians: a randomized trial. JAMA 2000; 283: 59-68. 